\title{
Direct evidence for symbiont sequestration in the marine red tide ciliate Mesodinium rubrum
}

\author{
P. J. Hansen ${ }^{1, *}$, M. Moldrup ${ }^{1}$, W. Tarangkoon ${ }^{1,2}$, L. Garcia-Cuetos ${ }^{3}$, Ø. Moestrup ${ }^{3}$ \\ ${ }^{1}$ Marine Biological Laboratory, Department of Biology, University of Copenhagen, Strandpromenaden 5, Helsingør 3000, \\ Denmark \\ ${ }^{2}$ Faculty of Science and Fisheries Technology, Rajamangala University of Technology Srivijaya, Trang 92150, Thailand \\ ${ }^{3}$ Phycology Laboratory, Department of Biology, University of Copenhagen, Øster Farimagsgade 2D, Copenhagen 1353, \\ Denmark
}

\begin{abstract}
The red tide ciliate Mesodinium rubrum (= Myrionecta rubra) is known to contain symbionts of cryptophyte origin. Molecular data have shown that the symbiont is closely related or similar to free-living species of the Teleaulax/Plagioselmis/Geminigera clade. This suggests that the symbiont of $M$. rubrum is either a temporary symbiont or a quite recently established symbiont. Here, we present data from a number of experiments in which we offered M. rubrum a phototrophic dinoflagellate and 8 different cryptophyte species belonging to 5 different clades. Mesodinium rubrum was only able to grow when fed the 2 cryptophyte species belonging to the genus Teleaulax, T. acuta and T. amphioxeia. Using the nucleomorph large subunit rDNA gene as marker, we were able to discriminate the 2 Teleaulax species, allowing monitoring of the exchange of the symbionts in M. rubrum. Over a period of $35 \mathrm{~d}$, M. rubrum was able to exchange its symbionts from $T$. amphioxeia symbionts to $T$. acuta symbionts. This research suggests that $M$. rubrum can only utilize prey within the Teleaulax/Plagioselmis/Geminigera clade for sustained high growth rates and provides the first time-frame of endosymbiont replacement by M. rubrum.
\end{abstract}

KEY WORDS: Mesodinium rubrum $\cdot$ Symbionts $\cdot$ Ingestion $\cdot$ Cryptophytes

\section{INTRODUCTION}

Mesodinium rubrum is a phototrophic ciliate that hosts cryptophyte symbionts consisting of chloroplasts, mitochondria, nucleomorphs, cytoplasm and a so-called 'symbiont nucleus' (Taylor et al. 1971, Hibberd 1977, Hansen \& Fenchel 2006). The ciliate is well known for its ability to form dense red tides worldwide (e.g. Taylor et al. 1971, Lindholm 1985), and it has recently been identified as prey for the diarrhetic shellfish poisoning (DSP)-causing dinoflagellates Dinophysis spp. (e.g. Park et al. 2006, Nishitani et al. 2008a,b, Riisgaard \& Hansen 2009), creating a renewal of interest within the scientific community.

Mesodinium rubrum is an obligate mixotroph requiring both food uptake and light for sustained growth. Currently, all isolates of M. rubrum main- tained in culture have been fed cryptophytes belonging to the genus Geminigera for the cold-water strain and Teleaulax for the all the temperate strains. Both genera are closely related and belong to the same clade, the Teleaulax/Plagioselmis/Geminigera (TPG) clade (Hoef-Emden 2008). Very little is known about the growth of $M$. rubrum on cryptophytes outside of the TPG clade. Recently, 2 Rhodomonas strains, CRMAL03 and CR-MAL06, have been tested as food for the Korean strain of $M$. rubrum, resulting in a very low M. rubrum growth rate only on CR-MAL03 (Park et al. 2007, Myung et al. 2011). However, M. rubrum obtains most of its carbon requirements through photosynthesis (Smith \& Hansen 2007). It only needs to ingest 1 cryptophyte cell (Teleaulax sp.) per day to grow at its maximum growth rate $\left(\sim 0.5 \mathrm{~d}^{-1}\right.$; Smith \& Hansen 2007). In terms of ingested carbon, the food 
uptake only accounts for 1 to $2 \%$ of the carbon requirements per day. Furthermore, $M$. rubrum is able to divide its chloroplasts when starved, and its symbionts can photoacclimate (Hansen \& Fenchel 2006, Moeller et al. 2011), indicating some genetic control of the symbionts. Because $M$. rubrum meets most of its carbon requirements by photosynthesis, the question arises why $M$. rubrum has to eat to sustain growth. One explanation could be that $M$. rubrum harbors permanent symbionts but needs to ingest prey to obtain some essential micronutrients. Another explanation could be a need for symbiont renewal when the symbionts have undergone a certain number of divisions within the host. This hypothesis implies that the chloroplasts have to be acquired at regular intervals for sustained growth. Previous molecular analyses of the nucleomorph and chloroplast genes of the chloroplasts of Mesodinium rubrum and its prey have revealed identical sequences for these 2 organisms in the 3 cultured strains of M. rubrum (Johnson et al. 2007, Park et al. 2007, Garcia-Cuetos et al. 2010), tending to agree with the latter hypothesis.

In 2010, Park et al. (2010) studied possible renewal of symbionts using 2 strains belonging to the TPG clade (CR-MAL01=Teleaulax amphioxeia and CRMAL11 = Teleaulax acuta; Park et al. 2010). A Mesodinium rubrum culture fed CR-MAL11 for 5 mo still contained chloroplast markers from CR-MAL01 (see Fig. 4 in Park et al. 2010). The aim of their study was the replacement of the chloroplasts in Dinophysis caudata, and the results obtained for M. rubrum were not discussed in detail. Some ambiguity, such as the extent of plastid renewal, was left unanswered. In a recent study, Myung et al. (2011) tried to study possible renewal of the plastids by feeding $M$. rubrum with a strain belonging to the Rhodomonas clade. They fed cryptophyte CR-MAL03 to a 2 mo starved $M$. rubrum culture for $2 \mathrm{~d}$ and found chloroplast markers of both the original prey item (CR-MAL01) and the subsequent prey item (CRMAL03) over a period of $37 \mathrm{~d}$ after the beginning of the experiment. The presence of both genetic markers makes it difficult to conclude whether there was full symbiont replacement and to what extent the chloroplasts inherited from CR-MAL03 were photosynthetically active.

To answer these questions, more information is needed concerning the growth performances of Mesodinium rubrum on a variety of cryptophyte strains belonging to different clades as well as the possible occurrence of symbiont turnover. The aim of the present investigation was to study the growth responses of the Danish isolate of $M$. rubrum when offered 8 different species of cryptophytes belonging to 5 different clades, in addition to one dinoflagellate species. The possible exchange of symbionts was examined using transmission electron microscopy (TEM) and molecular techniques.

\section{MATERIALS AND METHODS}

\section{Algal cultures}

A total of 8 species of cryptophytes and 1 species of dinoflagellate were used as food (Table 1). The selection represents a broad range of possible prey found in Mesodinium rubrum's natural environment. All cultures were grown on a glass table in h/20 medium (Guillard 1975) at a salinity of 30 and a temperature of $20 \pm 1^{\circ} \mathrm{C}$. Cool white light $(100 \mu \mathrm{mol}$ photons $\mathrm{m}^{-2} \mathrm{~s}^{-1}$ ) was provided from beneath in a light:dark cycle of 14:10 h.

\section{Cultures of the ciliate Mesodinium rubrum}

A culture of Mesodinium rubrum was established from single cells isolated from surface seawater samples collected in Frederikssund, Denmark, during a bloom event on April 17 2007, as described in Riisgaard \& Hansen (2009). Prior to the experiment, the cultures, normally grown in f/2 medium (Guillard 1975), were transferred to $\mathrm{h} / 20$ medium and acclimated for a couple of months. The change of medium was carried out to respond to the needs of certain cryptophytes species, such as Hanusia phi and Guillardia theta.

Table 1. Protist strains used as prey in the experiments. nmSSU (nSSU) rDNA: nucleomorph (nuclear) small subunit ribosomal DNA. na: not available

\begin{tabular}{|lccc|}
\hline \multirow{2}{*}{ Species } & Culture collection & \multicolumn{2}{c|}{ GenBank accession no. } \\
& ID no. & nmSSU rDNA & nSSU rDNA \\
\hline Heterocapsa rotundata & K-0483 (SCCAP) & na & na \\
Chroomonas vectensis & CCMP-432 & na & HM126534 \\
Guillardia theta & CCMP-2712 & AF083031 & X57162 \\
Hanusia phi & CCMP-325 & GQ396278 & U53126 \\
Hemiselmis tepida & CCMP-442 & GQ396279 & HM126533 \\
Proteomonas sulcata & CCMP-321 & na & HM126536 \\
Rhodomonas salina & K-1487 (SCCAP) & na & HM126532 \\
Teleaulax amphioxeia & K-0434 (SCCAP) & GQ396273 & AJ421146 \\
Teleaulax acuta & K-1486 (SCCAP) & HQ651177 & HM126531 \\
\hline
\end{tabular}




\section{Growth and survival responses of Mesodinium rubrum}

The 8 cryptophyte species and 1 dinoflagellate species were tested as prey for Mesodinium rubrum (Table 1). Initial attempts to culture Mesodinium rubrum on all 9 prey species, offered separately in multi-well dishes, revealed that long-term growth was only sustained with cryptophytes belonging to the genus Teleaulax, specifically $T$. acuta and $T$. amphioxeia.

To explore the relationship between Mesodinium rubrum and its prey, growth experiments were performed using all prey types. The experiments were carried out using $65 \mathrm{ml}$ tissue culture bottles filled to capacity. Samples (2 ml) were drawn at Days 4, 8, 12 (or 10) and in one case 16, and bottles were refilled to capacity with fresh h/20 medium. Samples were fixed in Lugol's fixative (final concentration $2 \%$ ), and cells were enumerated in a Sedgewick-Rafter chamber using an inverted microscope. Approximately one-tenth of the chamber was searched for organisms, leading to a detection limit of $\sim 10$ cells $\mathrm{m}^{-1}$.

It was previously shown that the growth of Mesodinium rubrum is affected when $\mathrm{pH}$ exceeds 8.5 and that $M$. rubrum dies if exposed to $\mathrm{pH}>8.8$ for days (Smith \& Hansen 2007). Thus, to interpret data correctly, pH was measured directly in the bottles on each sampling occasion using a Sentron ${ }^{\circledR}$ Argus $\mathrm{pH}$ meter equipped with a HOT-Fet line $\mathrm{pH}$ probe calibrated using standard buffers of $\mathrm{pH} 7$ and 10. Prior to all experiments, $M$. rubrum was grown on the cryptophyte Teleaulax amphioxeia. Only $M$. rubrum cultures that had just depleted the prey were used for experiments. Three sets of experiments with different prey items were carried out, making each session manageable in terms of manpower.

In the first set of experiments, the cryptophyte Teleaulax acuta was used as prey. Initial cell concentrations were 1000 cells $\mathrm{ml}^{-1}$ of both prey and predator. This prey concentration was chosen based on the previous observation that maximum growth of Mesodinium rubrum occurs during these conditions when fed T. amphioxeia. Moreover, it has been shown that $M$. rubrum ingests $\sim 1$ prey cell (T. amphioxeia) $\mathrm{d}^{-1}$ at this prey concentration (Smith \& Hansen 2007), which means that $M$. rubrum should be able to control the prey concentration in the bottles. In cases in which the prey was depleted in the experimental bottles, $M$. rubrum from that particular experiment was subcultured (i.e. diluted), and prey concentrations were increased and/or sampling frequency increased. Control experiments were carried out with a monoculture of unfed $M$. rubrum and monocultures of the given prey species. All experiments were carried out in triplicate.

In a second set of experiments, the cryptophyte Proteomonas sulcata and the dinoflagellate Heterocapsa rotundata were used as prey. In this set of experiments, Teleaulax amphioxeia was used a 'control food' to demonstrate the potential performance of the Mesodinium rubrum isolate.

Finally, in a third set of experiments, the growth responses of Mesodinium rubrum were studied when offered 5 additional species of cryptophytes: Chroomonas vectensis, Rhodomonas salina, Hanusia phi, Guillardia theta and Hemiselmis tepida. The setup of the experiment was the same as in the 2 previous sets of experiments, except that all the mixed cultures were diluted on Day 4 to diminish effects of elevated $\mathrm{pH}$ on the outcome of the experiments.

\section{DNA extraction of the cryptophytes and PCR amplification of nuclear SSU rDNA}

Prior to the growth experiments, DNA of the cryptophyte species used as prey (Table 1) was extracted as described in Hansen et al. (2003). PCR amplifications of the nuclear small subunit ribosomal DNA (nSSU rDNA) of the cryptophytes were completed in $50 \mu \mathrm{l}$ with a combination of primers CrN1F and SSUBR as outlined in Hoef-Emden et al. (2002). The nSSU rDNA gene was selected because it offers the biggest database in GenBank and allowed comparison between our strain and many other strains of cryptophytes.

\section{Symbiont sequestration: isolation of Mesodinium rubrum, PCR amplification and cloning of nmLSU rDNA}

During the grazing and growth experiments, samples were collected $(20 \mathrm{ml})$ and fixed in acid Lugol on Day 4 for all species tested as food for Mesodinium rubrum, on Day 12 for Hanusia phi, Hemiselmis tepida and Teleaulax acuta and on Days 16 and 35 for the experiment carried out with T. acuta. At the end of the experiments, the cells of $M$. rubrum from the experiments that sustained best growth were isolated for PCR: H. phi, H. tepida and T. acuta on Day 12 and cells of $M$. rubrum fed $T$. acuta on Days 16 and 35. In all cases, Lugol-fixed cells of $M$. rubrum were isolated using a drawn Pasteur glass pipette under an Olympus inverted microscope CKX31 (Olympus) and washed at least 3 times in 
$\mathrm{ddH}_{2} \mathrm{O}$ under the inverted microscope to prevent any cryptophyte present in the fixed sample from being transferred with the cell of interest. Finally, 5 washed $M$. rubrum cells were transferred into a single $0.2 \mathrm{ml}$ PCR tube (StarLab) and kept frozen at $-20^{\circ} \mathrm{C}$ until further processing.

PCR reactions were carried out in volumes of $50 \mu \mathrm{l}$. To determine the endosymbiont identity, PCR amplifications of the nucleomorph large subunit ribosomal DNA (nmLSU rDNA) were carried out as described by Garcia-Cuetos et al. (2010) with the primer combination nmLSUCr3F and D3B (Nunn et al. 1996). Subsequently, a semi-nested PCR amplification was performed using nmLSUCr3F and D2C (Scholin et al. 1994) and applying the same PCR profile. Ribosomal DNA genes are usually present within the telomeres of the 3 chromosomes of the nucleomorph (Douglas et al. 2001, Lane \& Archibald 2006, Lane et al. 2006) and are therefore easily amplified from a single or a few cells. Prior to amplification, physical disruption was conducted using glass beads (Sigma-Aldrich) to ensure rupture of the Mesodinium rubrum cells within the PCR tubes (Frommlet \& Iglesias-Rodríguez 2008). All PCR reactions were carried out on a PTC-200 Peltier Thermal Cycler (MJ Research).

To discriminate between possible copies of the nmLSU rDNA present in Mesodinium rubrum at different times during the experiment, all gene amplifications were cloned with the TOPO TA Cloning Kit (catalogue no. K4500-01) from Invitrogen ${ }^{\circledR}$. Following plating, transformed clones were selected, and the nmLSU rDNA was amplified, as described above.

\section{Purification and sequencing}

All DNA PCR products were purified using Nucleofast, following the manufacturer's recommendations (Macherry-Nagel). PCR product (500 ng) was airdried over night and sent to the sequencing service at Macrogen for determination in both directions using the same primers employed for amplification.

\section{Alignments and phylogenetic analyses}

$$
\text { nSSU rDNA alignment }
$$

To verify the phylogenetic position of the cryptophyte isolates within different marine clades and to confirm their morphological identification, a dataset with numerous cryptophyte taxa was analyzed, con- sisting of nSSU rDNA sequences. The sequences were first aligned using MAFFT 6.624 (Katoh \& Toh 2008 ) and then improved manually using BioEdit 7.0.5 sequence alignment software (Hall 1999). The dataset was composed of 5 new sequences and 46 sequences retrieved from GenBank. Four glaucophyte sequences were chosen as outgroup taxa based on previous phylogenetic studies of noncoding genes (Bhattacharya et al. 1995, Hoef-Emden 2008).

A Bayesian method was used to infer phylogeny, using the program MrBayes v.3.2 (Huelsenbeck \& Ronquist 2001). Two simultaneous Monte Carlo Markov chains (MCMC; Yang \& Rannala 1997) were run from random trees for a total of 2000000 generations (metropolis-coupled MCMC). One of every 50 trees was sampled. AWTY (Wilgenbusch et al. 2004) was used to graphically evaluate the extent of the MCMC analysis. After excluding the first sampled trees categorized as the 'burn-in period', a consensus tree was constructed using PAUP* 4.0.b10 software (Swofford 2002), based on 39000 trees. Modeltest (Posada \& Crandall 1998), implemented in the PAUP* 4.0.b10 software (Swofford 2002), identified the GTR model as the best. Using these settings, a tree was reconstructed with the online version of the PhyML software (Guindon \& Gascuel 2003; available on the Montpellier bioinformatics platform at www.atgc-montpellier.fr/phyml), and using the maximum likelihood (ML) method (Felsenstein 1981). The reliability of internal branches was assessed using the bootstrap method with 100 replicates (Felsenstein 1985).

\section{nmLSU rDNA alignment}

An alignment of the nmLSU rDNA sequences was completed to compare all clones from the cloning experiments with the sequences of the cryptophytes in GenBank (see accession numbers in Table 1). For the nSSU rDNA of cryptophyte alignment, the sequences were first aligned using MAFFT 6.624 (Katoh \& Toh 2008) and then improved manually using BioEdit 7.0.5 sequence alignment software.

\section{Transmission electron microscopy (TEM)}

Most species of cryptophytes have chloroplasts in which thylakoids are grouped in pairs (Hill 1991). Exceptions occur within the genera Teleaulax and Plagioselmis, where the thylakoids are grouped in 
triplets, as in Mesodinium rubrum (Hibberd 1977, Garcia-Cuetos et al. 2010). To investigate possible incorporation of chloroplasts from cryptophyte species not belonging to the TPG clade, a culture of Mesodinium rubrum fed Hemiselmis tepida was mixed 1:1 with $4 \%$ glutaraldehyde in $0.2 \mathrm{M}$ cacodylate buffer at $\mathrm{pH} 7.4$ and containing $0.4 \mathrm{M}$ sucrose. After $1 \mathrm{~h}$ at $4^{\circ} \mathrm{C}$, the cells were concentrated by centrifugation and rinsed twice in cold cacodylate buffer of decreasing sucrose content. Once rinsed, the material was post-fixed overnight in $2 \%$ osmium tetroxide in $0.2 \mathrm{M}$ cacodylate buffer at pH 7.4 and $4{ }^{\circ} \mathrm{C}$. Before dehydration, the material was rinsed briefly in buffer. Each step of the dehydration lasted $20 \mathrm{~min}$ at $4^{\circ} \mathrm{C}$ in the following ethanol concentrations: 30, 50, 70, 90 and $96 \%$. The material was transferred to room temperature while in $96 \%$ ethanol, and the dehydration was completed in 2 changes of absolute ethanol, $20 \mathrm{~min}$ in each change. Following 2 brief rinses in propylene oxide, the material was transferred to a 1:1 mixture of Spurr's embedding mixture (Spurr) and propylene oxide and left uncovered overnight, followed by $5 \mathrm{~h}$ in a fresh mixture of Spurr. The material was then moved to a new recipient, and Spurr was added. Finally, it was polymerized at $70^{\circ} \mathrm{C}$ overnight. Sectioning was carried out on a Reichert Ultracut E ultramicrotome using a diamond knife. The sections were collected on slot grids (Rowley \& Moran 1975) and stained for $15 \mathrm{~min}$ with $2 \%$ uranyl acetate in methanol, followed by Reynold's lead citrate. The grids were examined in a JEM-1010 electron microscope (JEOL), fitted with a Gatan 792 digital camera (Gatan).

\section{RESULTS}

\section{Phylogeny of cryptophytes used in the present experiment}

The nSSU rDNA alignment consisted of $1712 \mathrm{bp}$. The molecular phylogeny inferred from Bayesian analysis yielded the tree topology shown in Fig. 1. Glaucophytes rooted the tree, and the cryptophytes were divided into 5 clades. The first clade included 3 genera: Hemiselmis, Chroomonas and Komma. The second clade comprised 4 genera: Rhodomonas, Rhinomonas, Pyrenomonas and Storeatula. The third clade was formed by 2 genera: Hanusia and Guillardia, while the fourth clade was composed of Cryptomonas only. Finally, the fifth clade included 3 genera: Teleaulax, Plagioselmis and Geminigera. Within this clade, the genus Teleaulax appeared to be polyphyletic. Proteomonas sulcata and Falcomonas daucoides took up isolated positions and did not belong to any of the 5 clades. Thus, the species used included species from 5 different clades (see Fig. 1).

\section{Growth response of Mesodinium rubrum when fed Teleaulax acuta and $T$. amphioxeia as prey}

In the experiments in which Mesodinium rubrum was fed Teleaulax acuta or T. amphioxeia, M. rubrum grew in the unfed control experiments during the first 4 to $8 \mathrm{~d}$ of the incubation (Figs. 2A, 3A \& 4A, Table 2), but the growth rate leveled off. However, the control culture of $M$. rubrum used in the first set of experiments with $T$. acuta went through more cell divisions than those used in the 2 latter experiments (Figs. 3 \& 4, Table 2), indicating a better feeding status of $M$. rubrum in the former culture prior to the experiments.

When Mesodinium rubrum was fed Teleaulax acuta or T. amphioxeia at a prey:predator ratio of 1:1, 
the growth of $M$. rubrum was not significantly different from the unfed control during the first 8 or $4 \mathrm{~d}$, respectively. During these periods, the prey was depleted below the detection limit (Figs. 2B \& 3B).
When $M$. rubrum was re-fed $T$. amphioxeia at a prey predator ratio of 5:1 on Day 4, growth increased considerably during the subsequent $4 \mathrm{~d}$ of incubation (Fig. 3A), and the prey was again depleted below

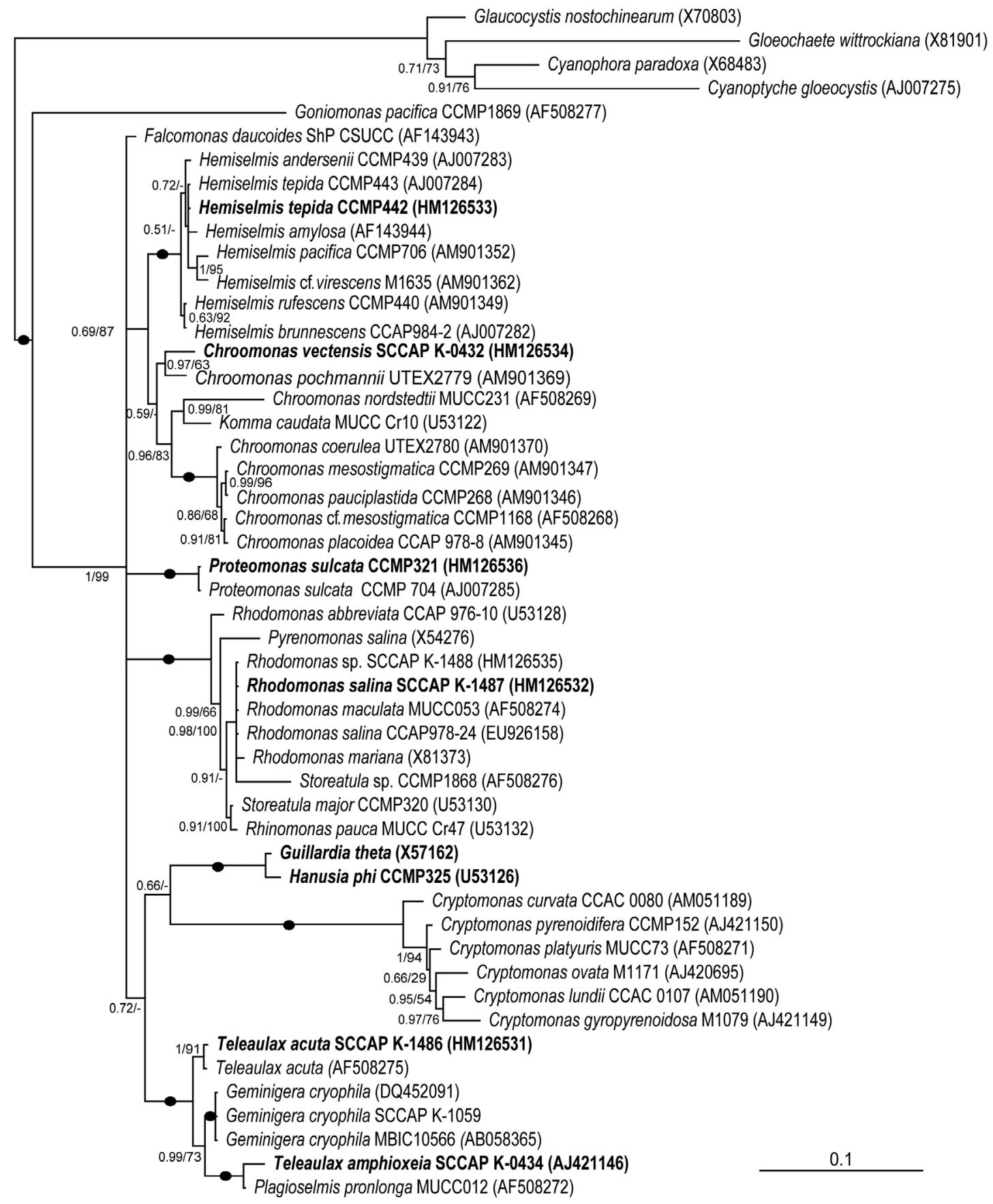

Fig. 1. Phylogeny of cryptophytes based on nuclear small subunit rDNA sequences (1712 bp) inferred from Bayesian analysis. Glaucocystis nostochinearum, Gloeochaete wittrockiana, Cyanophora paradoxa and Cyanoptyche gloeocystis were used as outgroups. Branch support was obtained from Bayesian posterior probabilities and bootstrap (100 replicates) in maximum likelihood (ML) analyses. GenBank accession numbers in parentheses. At the internodes, posterior probabilities (1) are written first, followed by bootstrap values (\%) from ML. (•) Highest possible posterior probability (1.0) and bootstrap value (100\%). Scale bar represents 10 substitutions per site. Species in bold face were used for the experiments in this study 

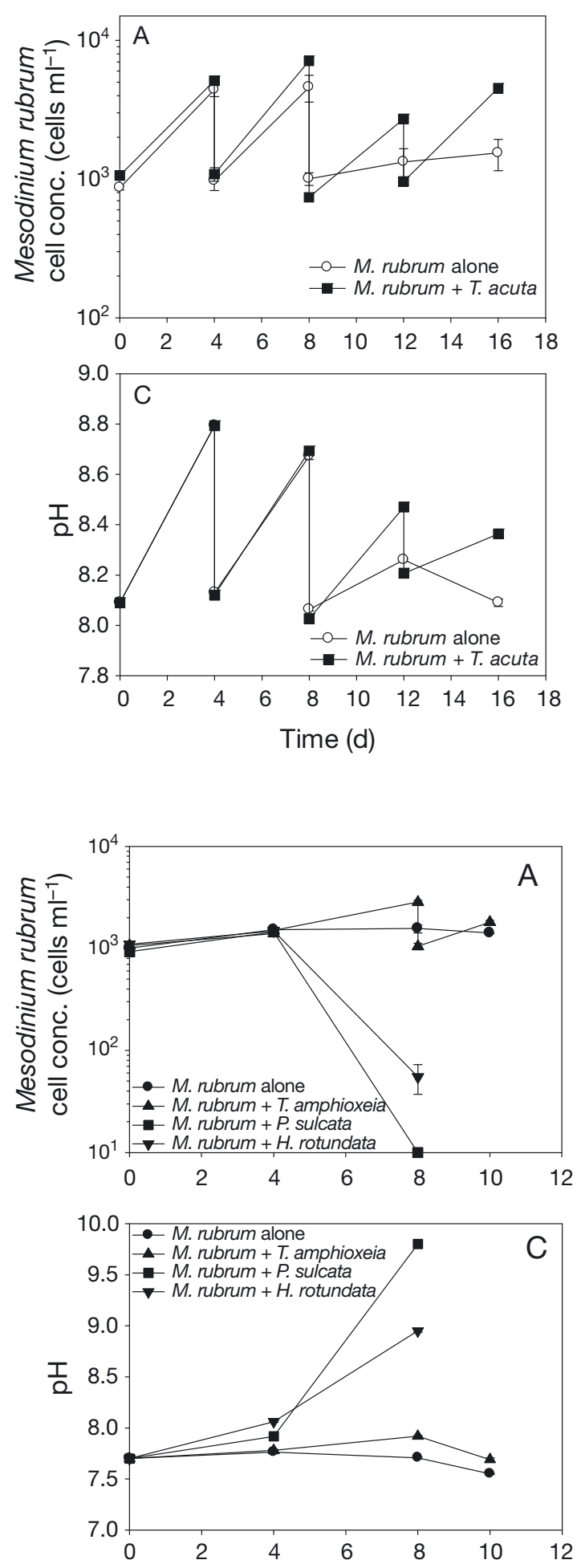

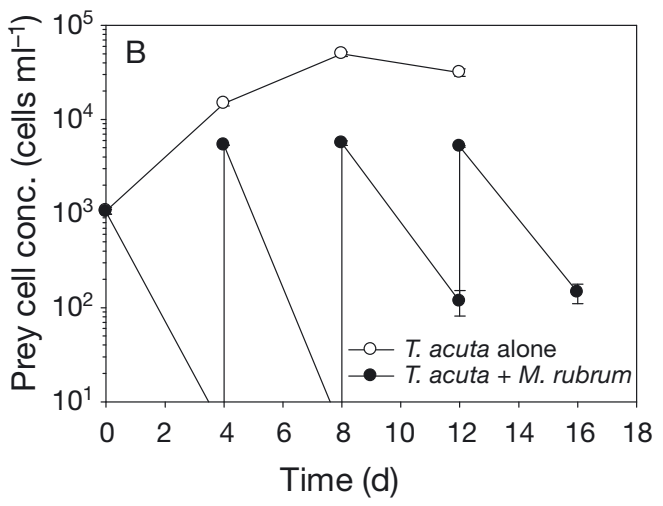

Fig. 2. Growth response Expt 1. (A) Changes of cell concentrations of Mesodinium rubrum as a function of incubation time (d) in monoculture and when grown in mixed cultures with the cryptophyte Teleaulax acuta. Drops in cell concentrations on Days 4, 8 and 12 are due to dilution of cultures to avoid effects of elevated $\mathrm{pH}$ on the ciliates. (B) Changes of prey cell concentrations in monocultures and in the mixed cultures with $M$. rubrum. Sudden increases of prey concentrations on Days 4, 8, and 12 are due to additions of prey. (C) Development of $\mathrm{pH}$ in the cultures. Drops of $\mathrm{pH}$ in the mixed cultures are due to dilutions and therefore additions of growth medium with lower $\mathrm{pH}$
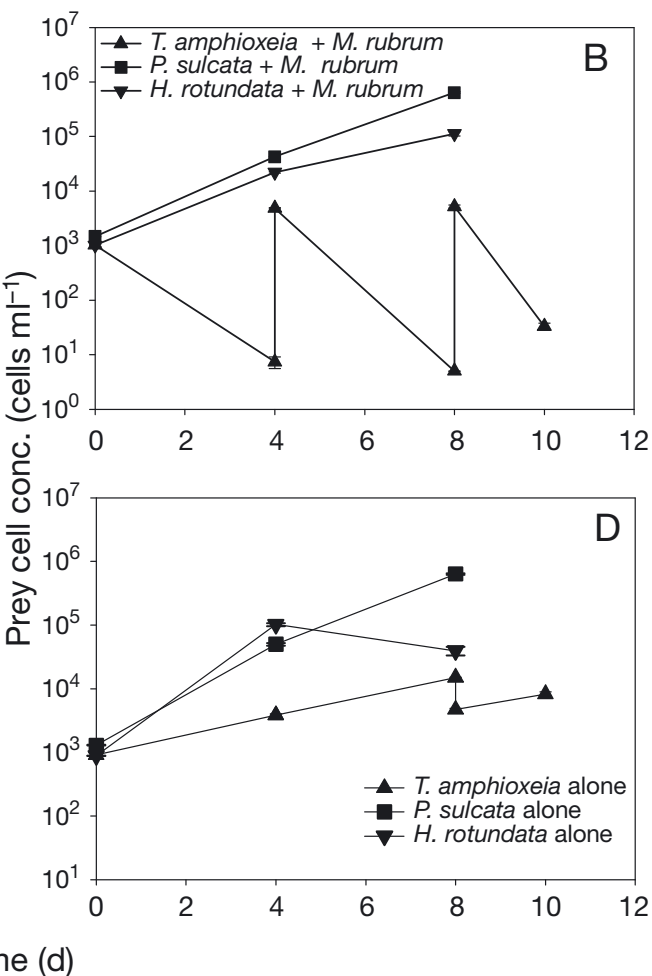

Time (d)

Fig. 3. Growth response Expt 2. (A) Changes of cell concentrations of Mesodinium rubrum as a function of incubation time (d) in monoculture and when grown in mixed cultures with the cryptophytes Teleaulax amphioxeia or Proteomonas sulcata or the dinoflagellate Heterocapsa rotundata. Drops in cell concentrations on Day 8 in the mixed culture of $M$. rubrum $+T$. amphioxeia are due to dilution. (B) Changes of prey cell concentrations in the mixed cultures. Sudden increases in prey concentrations on Days 4 and 8 are due to additions of prey. (C) Development of $\mathrm{pH}$ in the cultures. (D) Changes of prey cell concentrations in monocultures. Detection limit was $\sim 10$ cells $\mathrm{ml}^{-1}$ 

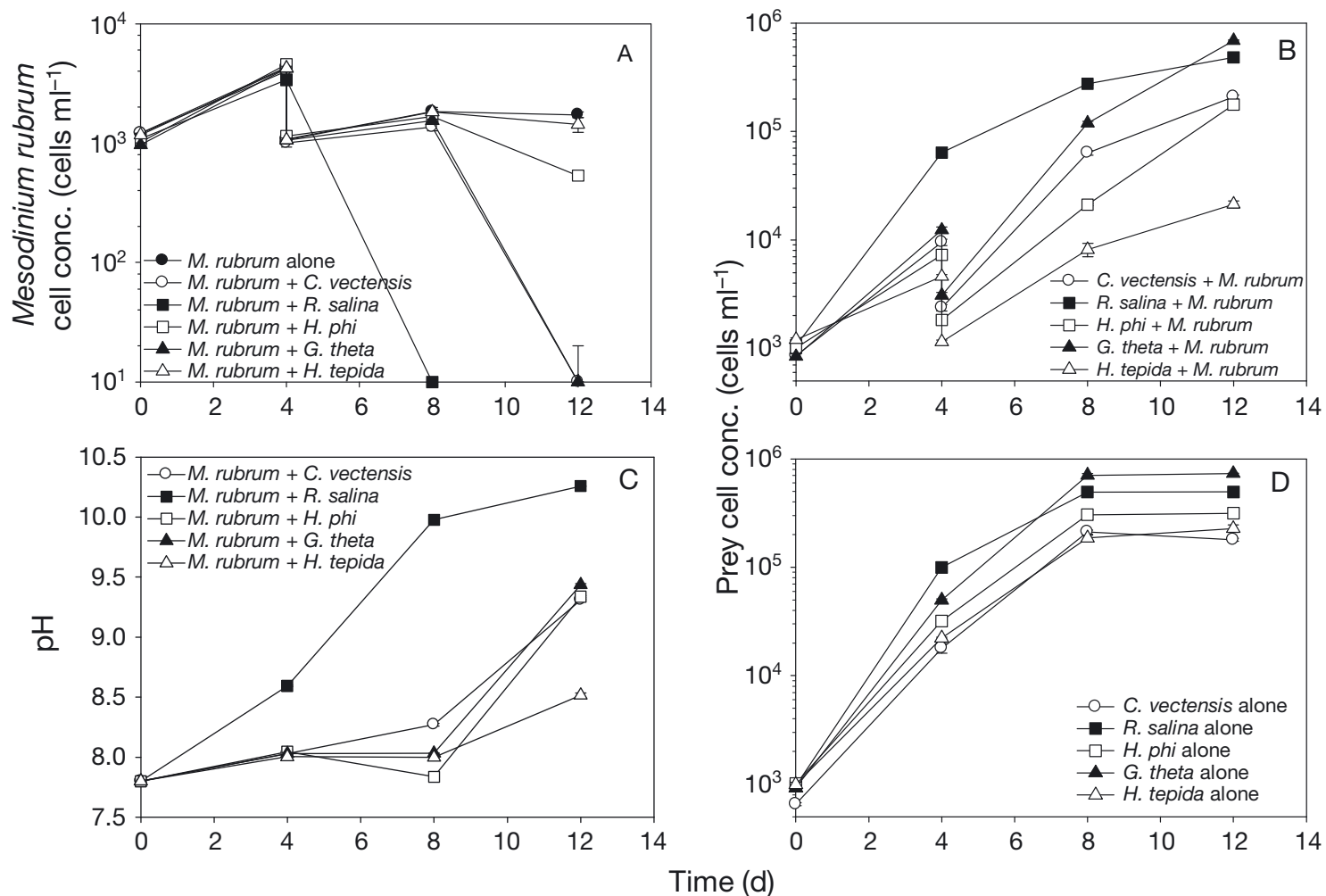

Fig. 4. Growth response experiment 3. (A) Changes of cell concentrations of Mesodinium rubrum as a function of incubation time (d) in monoculture and when grown in mixed cultures with the cryptophytes Chroomonas vectensis, Rhodomonas salina, Hanusia phi, Guillardia theta and Hemiselmis tepida. Drops in cell concentrations on Day 4 are due to dilution. (B) Changes of prey cell concentrations in the mixed cultures. Drops in prey cell concentrations on Day 4 are due to dilution of cultures. (C) Development of $\mathrm{pH}$ in the cultures. (D) Changes of prey cell concentrations in monocultures. Detection limit was $\sim 10 \mathrm{cells}^{-1}$

detection limit (Fig. 3B). A second re-feeding of the M. rubrum on Day 8, in combination with a shortening of the incubation period to $2 \mathrm{~d}$, resulted in a further increase in the M. rubrum growth rate (Fig. 3A). However, in both cases, the prey was not completely depleted on Day 10 in the experiments with $T$. amphioxeia or on Day 12 and 16 in the experiments with $T$. acuta (Figs. 2B \& 3B). The growth of $T$. acuta and $T$. amphioxeia in monoculture was studied for comparison (Figs. 2B \& 3D, Table 2).

\section{Growth responses of Mesodinium rubrum when fed organisms not belonging to the TPG clade}

Mesodinium rubrum could not sustain growth when fed the dinoflagellate Heterocapsa rotundata and the 5 non-TPG clade cryptophyte species tested (Figs. 3A \& 4A). Initial growth of $M$. rubrum in the mixed cultures containing these prey types was observed for the first 4 to $8 \mathrm{~d}$, but the growth rate never exceeded the growth of $M$. rubrum in monoculture. In some cases, $M$. rubrum died in the mixed cultures between
Day 4 and Day 12, depending on the prey species (Figs. 3A \& 4A). The death of M. rubrum always coincided with an increase in $\mathrm{pH}$ above 9 (Figs. 3C \& 4C).

In many cases, the prey items grew fast in both the algal monocultures and the mixed cultures (Figs. 3B,D \& 4B,D). The $\mathrm{pH}$ increased above 9 after 8 to $12 \mathrm{~d}$ of incubation, and growth of the algae then decreased or stopped. A comparison of the growth response of the prey in the monocultures and in the mixed cultures with Mesodinium rubrum during the first $4 \mathrm{~d}$ of the incubation revealed that the prey concentrations in the mixed cultures were always lower than in the monocultures.

\section{Sequestration of cryptophyte symbionts by Mesodinium rubrum}

The nmLSU rDNA provided a very precise molecular signature for the plastids of the different cryptophyte species when symbiont replacement was tested (see estimated divergence between sequences in Table 3). The alignment consisted of 
Table 3. Estimated evolutionary divergence (\%) between nucleomorph large subunit rDNA sequences of cryptophytes

\begin{tabular}{|lccccc|}
\hline Species & G. theta & H. phi & H. tepida & T. amphioxeia & T. acuta \\
\hline Guillardia theta & 0.00 & & & & \\
Hanusia phi & 9.7 & 0.00 & & & \\
Hemiselmis tepida & 31 & 30.5 & 0.00 & & \\
Teleaulax amphioxeia & 31 & 29.4 & 23.9 & 0.00 & \multirow{2}{*}{0.00} \\
Teleaulax acuta & 30 & 29.1 & 24.2 & 17.4 & \\
\hline
\end{tabular}

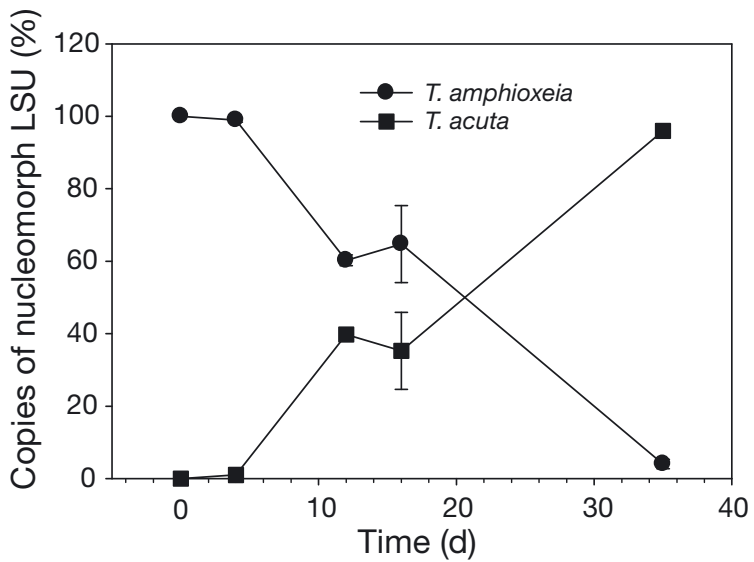

Fig. 5. Mesodinium rubrum. Symbiont exchange experiment. The percentage of symbiont nucleomorph large subunit (LSU) copies in an experiment in which prey was switched from Teleaulax amphioxeia to T. acuta at the start of the experiment. Values are treatment means $\pm \mathrm{SE}, \mathrm{n}=4$, except for at Day 35, where $\mathrm{n}=3$

$360 \mathrm{bp}$. When compared to the sequences retrieved from GenBank (Table 1), all of the sequences retrieved after cloning were identical to Teleaulax amphioxeia (GQ396273) except when Mesodinium rubrum was fed T. acuta. A total of 60 to 70 sequences were retrieved per cloning experiment replicate. When $M$. rubrum was fed $T$. acuta, the percentage of sequences belonging to $T$. amphioxeia retrieved from $M$. rubrum decreased, while that of $T$. acuta increased as the experiment went on (Fig. 5). However, the replacement rate appeared to be slow. After 15 to $18 \mathrm{~d}$ of exposure to T. acuta, only about half of the sequences retrieved from $M$. rubrum belonged to $T$. acuta. After $35 \mathrm{~d}$ of exposure to $T$. acuta, about $94 \%$ of the sequences retrieved from $M$. rubrum derived from $T$. acuta.

\section{Transmission electron microscopy studies}

A series of micrographs obtained from a culture of Mesodinium rubrum fed the cryptomonad Hemi- selmis tepida are shown in Fig. 6. There are several chloroplasts in the cell (Fig. 6A), located in both the oral and the aboral end of the cell. Collectively, we designate these as chromatophores because their origins differ (see below). Hemiselmis is ingested at the oral end of Mesodinium, which here possesses, in addition to a small cytostome, bands of microtubules and a number of tentacles (Fig. 6B). Hemiselmis cells are ingested whole (Fig. 6C,D), and Fig. 6C illustrates a cell in which the cryptomonad nuclei, the cryptomonad chloroplasts and the 2 types of trichocysts (ejectosomes) of Hemiselmis are visible. One of the 2 macronuclei of Mesodinium is visible in Fig. 6A, while the small nucleus in the upper part of the cell is from a prey cell. Mesodinium has 2 types of chromatophores (Fig. 6E,F). In one type, the permanent chromatophore or chloroplast, the thylakoids are grouped in lamellae of 3 thylakoids (Fig. 6E). For comparison, the chloroplasts from Hemiselmis located within Mesodinium showed the 2-thylakoid lamellae characteristic of Hemiselmis (Fig. 6F). In contrast to the 3thylakoid lamellae, the 2-thylakoid lamellae of Hemiselmis were often not well preserved in the sections, indicating that they were being subjected to digestion enzymes.

\section{DISCUSSION}

\section{Growth responses of Mesodinium rubrum when fed different types of prey}

The present results suggest that the Danish strain of Mesodinium rubrum could only sustain growth for at least $12 \mathrm{~d}$ when supplied with Teleaulax acuta and T. amphioxeia as prey. Park et al. (2007) studied the growth response of starved $M$. rubrum (Korean strain MR-MAL01) when offered 4 cryptophytes belonging to the TPG clade and 2 belonging to the 'Rhodomonas clade' (CR-MAL03 and CR-MAL06). Except for CR-MAL06, positive growth rates were observed during the incubation time studied (6 d), and all rates were significantly different from the unfed control. The results indicate that MR-MAL01 can grow on species not closely related to Teleaulax, although the observed growth rate on the CR-MAL03 strain was low $\left(\mu=0.16 \mathrm{~d}^{-1}\right)$. This observation was confirmed by Myung et al. (2011), who found low growth rates of the same strain of $M$. rubrum when starved cells 


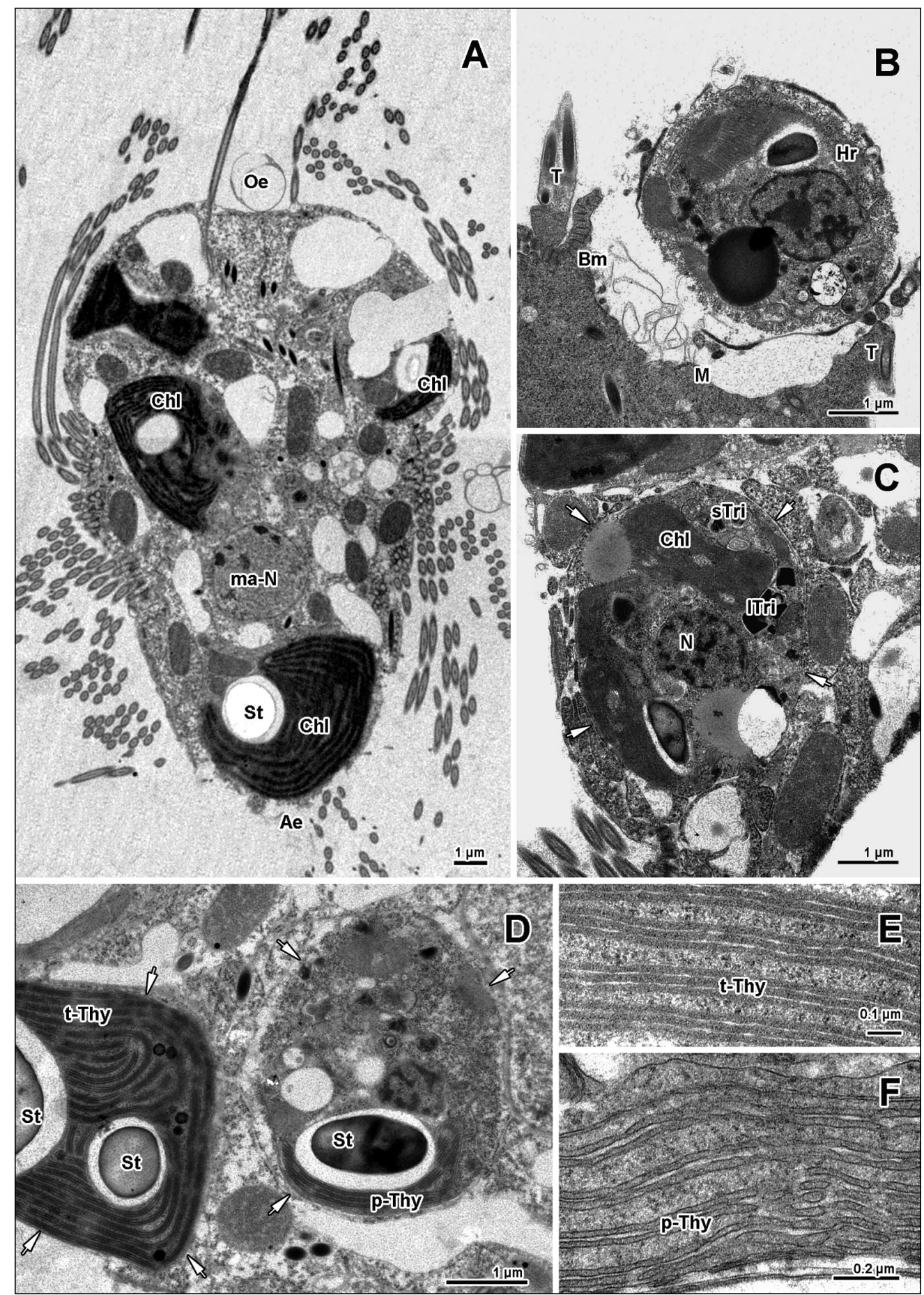

Fig. 6. Mesodinium rubrum grown in culture and fed Hemiselmis tepida. (A) Longitudinal section of $M$. rubrum from the oral end (Oe) to the aboral end (Ae) illustrating the cilia, chloroplasts (Chl), starch grains (St), macronucleus (ma-N) and the bands of microtubules around the mouth. (B) The mouth (M) of $M$. rubrum using the tentacles (T) to engulf a cell of $H$. tepida (Hr) and showing some of the bands of microtubules (Bm). (C) Engulfed cell of $H$. tepida (arrows) within M. rubrum. Visible organelles are the chloroplast, the small (sTri) and large trichocysts (lTri) and the cryptomonad nucleus (N). (D) Chloroplast of M. rubrum (arrowheads) with triplets of thylakoids (t-Thy) next to an engulfed cell of $H$. tepida (arrows) with chloroplast showing paired thylakoids (p-Thy). (E) Detail of the chloroplast of M. rubrum with thylakoids in triplets. (F) Detail of the chloroplast of $H$. tepida, with paired thylakoids 
were fed CR-MAL03 over a longer period (10 d). Ingestion rates were not provided, but the data presented by Myung et al. (2011) indicate ingestion. $M$. rubrum was not able to control the prey populations in any of these studies, which is in agreement with our present observations.

The Danish isolate of Mesodinium rubrum was unable to grow on cryptophytes outside the TPG clade. However, the strain of Rhodomonas salina presently used as food in our studies is not identical to CR-MAL03 or CR-MAL06, and thus the possibility that the Danish $M$. rubrum strain can grow on another Rhodomonas species cannot be entirely ruled out.

However, no matter the strain of Mesodinium rubrum, it appears that cryptophytes within the TPG clade support the growth of M. rubrum significantly better than cryptophytes of other clades. Presently, all cultures are maintained on cryptophytes from this clade (e.g. Gustafson et al. 2000, Johnson \& Stoecker 2005, Johnson et al. 2006, Park et al. 2007, the present study; Table 4). One possible explanation could be that $M$. rubrum relies on regular symbiont sequestration for sustained growth and that species within the TPG clade function best as symbionts.

A comparison of the growth response of the prey in monocultures and in the mixed cultures with Mesodinium rubrum during the first $4 \mathrm{~d}$ of the incubation revealed that prey concentrations in the mixed cultures were always lower than in the monocultures, indicating that M. rubrum ingested all types of the offered prey items (Table 2). However, the design of the growth experiments did not allow for estimations of ingestion rates due to the low sampling frequency (typically every $4 \mathrm{~d}$ ) and the large change in prey

Table 4. List of Mesodinium rubrum cultures and prey that the culture can be maintained on

\begin{tabular}{|c|c|c|}
\hline $\begin{array}{l}\text { Place of origin } \\
\text { of culture }\end{array}$ & Prey & Source \\
\hline Antarctica & Geminigera cryophila & $\begin{array}{l}\text { Gustafson et al. (2000), } \\
\text { Johnson \& Stoecker (2005), } \\
\text { Johnson et al. (2006, 2007), } \\
\text { Hackett et al. (2009) }\end{array}$ \\
\hline Denmark & $\begin{array}{l}\text { Teleaulax sp. } \\
\text { Teleaulax amphioxeia } \\
\text { T. acuta and T. amphioxeia }\end{array}$ & $\begin{array}{l}\text { Hansen \& Fenchel (2006), } \\
\text { Smith \& Hansen (2007) } \\
\text { Riisgaard \& Hansen (2009) } \\
\text { Present study }\end{array}$ \\
\hline Korea & $\begin{array}{l}\text { Teleaulax spp., unidentified } \\
\text { strains of the TPG clade }\end{array}$ & $\begin{array}{l}\text { Yih et al. }(2004), \text { Park et al. } \\
(2006,2007,2010)\end{array}$ \\
\hline Japan & Teleaulax amphioxeia & $\begin{array}{l}\text { Nagai et al. 2008, Nishitani } \\
\text { et al. }(2008 a, b)\end{array}$ \\
\hline
\end{tabular}

populations, which allow many types of possible interactions (e.g. nutrient limitation, allelopathy, etc.) between the prey and M. rubrum.

\section{Does Mesodinium rubrum sequester and replace chloroplasts via prey ingestion?}

Using the nmLSU rDNA gene, we were able to demonstrate a gradual shift from the Teleaulax amphioxeia to the T. acuta chloroplast type when the diet of Mesodinium rubrum was changed from $T$. amphioxeia to T. acuta, proving that replacement of chloroplasts took place. The replacement of the chloroplasts was relatively slow ( $>35 \mathrm{~d}$ for full replacement), indicating that only 'low performance' chloroplasts are renewed and that division of the chloroplast occurs within the cell. These results agree with the preliminary data published by Park et al. (2010) and provide for the first time a symbiont renewal time frame. In a previous study, the Danish strain of M. rubrum was believed to harbor a permanent cryptophyte endosymbiont due to the ability of the ciliate to duplicate its chloroplasts and the symbiont nucleus when starved of prey (Hansen \& Fenchel 2006). The present results clearly show that the Danish strains of $M$. rubrum acquire symbiont chloroplasts from prey via ingestion, just like the Korean and Antarctic strains of M. rubrum.

Although Mesodinium rubrum cultures could not sustain growth in the long run, when fed Hanusia phi and Hemiselmis tepida, the cultures did survive for at least $12 \mathrm{~d}$, which is sufficient time for chloroplast replacement to occur, based on the data collected from the experiment carried out with Teleaulax acuta and T. amphioxeia. The molecular experiment used to detect the change of plastids was therefore repeated for $H$. phi and H. tepida. However, we found no evidence of plastid replacement. To ensure that no plastids were present in low concentrations in the ciliate or missed due to primer bias, TEM sections of $M$. rubrum exposed to $H$. tepida were studied in detail. Prey cells taken up by $M$. rubrum were located in food vacuoles and often partly digested. In contrast to the normal endosymbionts of $M$. rubrum, which comprised plastids and mitochondria of cryptophyte origin in addition to a symbiont nucleus, only whole cells of $H$. tepida were 
observed. Thus, we found no proof of chloroplast sequestration by M. rubrum when fed $H$. tepida.

Recently, the Korean isolate of Mesodinium rubrum was shown to contain plastids from 2 different clades. Cultures originally grown on a Teleaulax species (CR-MAL01) were exposed to a Rhodomonas species (CR-MAL03) for $2 \mathrm{~d}$, after which the prey was removed. No complete exchange from the CRMAL01 to the CR-MAL03 genetic marker was detected, but signals from both markers were maintained for at least $14 \mathrm{~d}$ without prey, suggesting that the chloroplasts were actually sequestered (Myung et al. 2011). The lack of a complete chloroplast turnover may perhaps be explained by the short exposure to the new cryptophyte prey. Long-term growth of $M$. rubrum on CR-MAL03 may be necessary to fully exchange chloroplasts, as observed in the present study. It therefore remains unclear whether the Korean or other isolates of M. rubrum can in the long term grow on cryptophytes not belonging to the TPG clade and completely exchange its symbionts.

Johnson et al. (2007) found evidence that the Antarctic strain of Mesodinium rubrum is able to sequester not only chloroplasts from its prey but also cryptophyte nuclei. Nuclei were retained for up to $30 \mathrm{~d}$ in this strain of $M$. rubrum, and the nuclei were transcriptionally active and apparently able to service plastids derived from multiple cryptophyte cells. Johnson et al. (2007) suggested that the symbiont nucleus of $M$. rubrum derived from ingested prey is necessary to control the activity of the plastids and their duplication. However, whether the symbiont nucleus regulates plastid activity is still unknown. To what extent the Danish strains of M. rubrum depends upon sequestration of nuclear material from its prey is currently unknown. The fate of the symbiont nuclei within M. rubrum, when prey is switched from one prey species to another, is likewise unknown. Therefore, future experiments should address the exact role of the symbiont nucleus and how the ciliates control the functioning and division of chloroplasts from different prey species.

Acknowledgements. This study was supported by a grant from the Danish Research Council to P.J.H. (grant no 27206-0485). PhD grants from the University of Copenhagen supported M.M. and L.G.-C., while a PhD grant from the Rajamangala University of Technology, Srivijaya, Thailand, supported W.T.

\section{LITERATURE CITED}

Bhattacharya D, Helmchen T, Bibeau C (1995) Comparisons of nuclear-encoded small subunit ribosomal RNAs reveal the evolutionary position of the Glaucocystophyta. Mol Biol Evol 12:415-420

> Douglas S, Zauner S, Fraunholz M, Beaton M and others (2001) The highly reduced genome of an enslaved algal nucleus. Nature 410:1091-1096

Felsenstein J (1981) Evolutionary trees from DNA sequences: a maximum likelihood approach. J Mol Evol 17: 368-376

Felsenstein J (1985) Confidence limits on phylogenies: an approach using the bootstrap. Evolution 39:783-791

Frommlet J, Iglesias-Rodríguez D (2008) Microsatellite genotyping of single cells of the dinoflagellate species Lingulodinium polyedrum (Dinophyceae): a novel approach for marine microbial population studies. J Phycol 44:1116-1125

Garcia-Cuetos L, Moestrup Ø, Hansen PJ, Daugbjerg N (2010) The toxic dinoflagellate Dinophysis acuminata harbors permanent chloroplasts of cryptomonad origin, not kleptochloroplasts. Harmful Algae 9:25-38

$>$ Guindon S, Gascuel O (2003) A simple, fast, and accurate algorithm to estimate large phylogenies by maximum likelihood. Syst Biol 52:696-704

Guillard RRL (1975) Culture of phytoplankton for feeding marine invertebrates. In: WL Smith WL, Chanley MH (eds) Culture of marine invertebrate animals. Plenum Pub, New York, NY, p 29-60

Gustafson DE Jr, Stoecker DK, Johnson MD, Van Heukelem WF, Sneider K (2000) Cryptophyte algae are robbed of their organelles by the marine ciliate Mesodinium rubrum. Nature 405:1049-1052

Hackett JD, Mengmong T, Kulis DM, Fux E, Hess P, Bire R, Anderson DM (2009) DSP toxin production de novo in cultures of Dinophysis acuminata (Dinophyceae) from North America. Harmful Algae 8:873-879

Hall TA (1999) Bioedit: a user friendly biological sequence alignment editor and analysis program from windows 95/97/NT. Nucleic Acids Symp Ser 41:95-98

> Hansen PJ, Fenchel T (2006) The bloom-forming ciliate Mesodinium rubrum harbors a single permanent endosymbiont. Mar Biol Res 2:169-177

- Hansen G, Daugbjerg N, Franco JM (2003) Morphology, toxin composition and LSU rDNA phylogeny of Alexandrium minutum (Dinophyceae) from Denmark, with some morphological observations on other European strains. Harmful Algae 2:317-335

> Hibberd DJ (1977) Observation on ultrastructure of cryptomonad endosymbiont of red-water ciliate Mesodinium rubrum. J Mar Biol Ass UK 57:45-61

Hill DRA (1991) The systematic of cryptomonads. In: Patterson DJ, Larsen J (eds) Biology of free-living heterotrophic flagellates. Syst Assoc Spec 45:235-240

- Hoef-Emden K (2008) Molecular phylogeny of phycocyanincontaining cryptophytes: evolution of biliproteins and geographic distribution. J Phycol 44:985-993

> Hoef-Emden K, Marin B, Melkonian M (2002) Nuclear and nucleomorph SSU rDNA phylogeny in the Cryptophyta and the evolution of cryptophyte diversity. J Mol Evol 55: 161-179

Huelsenbeck JP, Ronquist F (2001) MRBAYES: Bayesian inference of phylogenetic trees. Bioinformatics 17:754-755

> Johnson MD, Stoecker DK (2005) Role of feeding in growth and photophysiology of Myrionecta rubra. Aquat Microb Ecol 39:303-312

Johnson MD, Tengs T, Oldach D, Stoecker DK (2006) Sequestration, performance, and functional control of 
cryptophyte plastids in the ciliate Myrionecta rubra (Ciliophora). J Phycol 42:1235-1246

Johnson MD, Oldach D, Delwiche CF, Stoecker DK (2007) Retention of transcriptionally active cryptophyte nuclei by the ciliate Myrionecta rubra. Nature 445:426-428

Katoh K, Toh H (2008) Recent developments in the MAFFT multiple sequence alignment program. Brief Bioinform 9: 286-298

Lane CE, Archibald JM (2006) Novel nucleomorph genome architecture in the cryptomonad genus Hemiselmis. J Eukaryot Microbiol 53:515-521

> Lane CE, Khan H, MacKinnon M, Fong A, Theophilou S, Archibald JM (2006) Insight into the diversity and evolution of the cryptomonad nucleomorph genome. Mol Biol Evol 23:856-865

Lindholm T (1985) Mesodinium rubrum-a unique photosynthetic ciliate. Adv Aquat Microb 3:1-48

> Moeller HV, Johnson MD, Falkowski PG (2011) Photoacclimation in the phototrophic ciliate Mesodinium rubrum (Ciliophora). J Phycol 47:324-332

Myung G, Kim HS, Park JS, Park MG, Yih W (2011) Population growth and plastid type of Myrionecta rubra depend on the kinds of available cryptomonad prey. Harmful Algae 10:536-541

> Nagai S, Nishitani G, Tomaru Y, Sakiyama S, Kamiyama T (2008) Predation by the toxic dinoflagellate Dinophysis fortii on the ciliate Myrionecta rubra and observation of sequestration of ciliate chloroplasts. J Phycol 44:909-922

Nishitani G, Nagai S, Sakiyama S, Kamiyama T (2008a) Successful cultivation of the toxic dinoflagellate Dinophysis caudata (Dinophyceae). Plankton Benthos Res 3:78-85

Nishitani G, Nagai S, Takano Y, Sakiyama S, Baba K, Kamiyama T (2008b) Growth characteristics and phylogenetic analysis of the marine dinoflagellate Dinophysis infundibulus (Dinophyceae). Aquat Microb Ecol 52: 209-221

Nunn GB, Theisen BF, Christensen B, Arctander P (1996) Simplicity-correlated size growth of the nuclear 28S ribosomal RNA D3 expansion segment in the crustacean order Isopoda. J Mol Evol 42:211-223

Park MG, Kim S, Kim HS, Myung G, Kang YG, Yih W (2006) First successful culture of the marine dinoflagellate Dinophysis acuminata. Aquat Microb Ecol 45:101-106
Park JS, Myung G, Kim HS, Cho BC, Yih W (2007) Growth responses of the marine photosynthetic ciliate Myrionecta rubra to different cryptomonad strains. Aquat Microb Ecol 48:83-90

> Park MG, Kim M, Kim S, Yih W (2010) Does Dinophysis caudata (Dinophyceae) have permanent plastids? J Phycol 46:236-242

> Posada D, Crandall KA (1998) MODELTEST: testing the model of DNA substitution. Bioinformatics 14:817-818

> Riisgaard K, Hansen PJ (2009) Role of food uptake for photosynthesis, growth and survival of the mixotrophic dinoflagellate Dinophysis acuminata. Mar Ecol Prog Ser 381:51-62

Rowley JC, Moran DT (1975) Simple procedure for mounting wrinkle-free sections on formvar-coated slot grids. Ultramicroscopy 1:151-155

> Scholin CA, Herzog M, Sogin M, Anderson DM (1994) Identification of group and strain-specific genetic markers for globally distributed Alexandrium (Dinophyceae). II. Sequence analysis of a fragment of the LSU rRNA gene. J Phycol 30:999-1011

Smith M, Hansen PJ (2007) Interaction between Mesodinium rubrum and its prey: importance of irradiance, prey concentration, and pH. Mar Ecol Prog Ser 338: $61-70$

Swofford DL (2002). PAUP* 4.0: phylogenetic analysis using parsimony (*and other methods). Sinauer Associates, Sunderland, MA

Taylor FJR, Blackbourn DJ, Blackbourn J (1971) The redwater ciliate Mesodinium rubrum and its 'incomplete symbionts': a review including new ultrastructural observations. J Fish Res Board Can 28:391-407

Wilgenbusch JC, Warren DL, Swofford DL (2004) AWTY: a system for graphical exploration of MCMC convergence in Bayesian phylogenetic inference. Available at http:// ceb.csit.fsu.edu/awty/

> Yang Z, Rannala B (1997) Bayesian phylogenetic inference using DNA sequences: a Markov chain Monte Carlo method. Mol Biol Evol 14:717-724

> Yih W, Kim HS, Jeong HJ, Myung G, Kim YG (2004) Ingestion of cryptophyte cells by the marine photosynthetic ciliate Mesodinium rubrum. Aquat Microb Ecol 36: $165-170$

Submitted: August 8, 2011; Accepted: January 23, 2012 Proofs received from author(s): March 5, 2012
Editorial responsibility: Robert Sanders,

Philadelphia, Philadelphia, USA 